\title{
Multiscale Vessel Enhancement Filtering ${ }^{\star}$
}

\author{
Alejandro F. Frangi, Wiro J. Niessen, Koen L. Vincken, Max A. Viergever \\ Image Sciences Institute, Utrecht University Hospital \\ Room E.01.334, Heidelberglaan 100, 3584 CX Utrecht, the Netherlands \\ \{alex, wiro, koen, $\max \}$ isi.uu.n1
}

\begin{abstract}
The multiscale second order local structure of an image (Hessian) is examined with the purpose of developing a vessel enhancement filter. A vesselness measure is obtained on the basis of all cigenvalues of the Hessian. This measure is tested on two dimensional DSA and three dimensional aortoiliac and cerebral MRA data. Its clinical utility is shown by the simultaneous noise and background suppression and vessel enhancement in maximum intensity projections and volumetric displays.
\end{abstract}

\section{Introduction}

Accurate visualization and quantification of the human vasculature is an important prerequisite for a number of clinical procedures. Grading of stenoses is important in the diagnosis of the severity of vascular disease since it determines the treatment therapy. Interventional procedures such as the placement of a prosthesis in order to prevent aneurysm rupture or a bypass operation require an accurate insight into the three dimensional vessel architecture.

Both two-dimensional projection techniques, such as DSA, and three-dimensional modalities as X-ray rotational angiography, CTA and MRA are employed in clinical practice. Although CTA and MRA provide volumetric data, the common way of interpreting these images is by using a maximum intensity projection.

The main drawbacks of maximum intensity projections are the overlap of non-vascular structures and the fact that small vessels with low contrast are hardly visible. This has been a main limitation in time-of-flight MRA [4]. In contrast enhanced MRA [12] the delineation of these vessels is considerably improved, but other organs can be still projected over the arteries.

The purpose of this paper is to enhance vessel structures with the eventual goal of vessel segmentation. A vessel enhancement procedure as a preprocessing step for maximum intensity projection display will improve small vessel delineation and reduce organ overprojection. Segmentation of the vascular tree will facilitate volumetric display and will enable quantitative measurements of vascular morphology.

There are several approaches to vessel enhancement. Some of them work at a fixed scale and use (nonlinear) combinations of finite difference operators applied in a set of orientations [2-4]. Orkisz et al. [11] presents a method that applies a median filter in the direction of the vessel. All these methods have shown problems to detect vessels over a large size range since they perform a fixed scale analysis. Moreover, to handle voxel anisotropy, these methods usually need to resample the dataset or to resource to $2 \frac{1}{2} D$ processing (cf. [11]). Multi-scale approaches to vessel enhancement include "cores" [1], steerable filters $[7,8]$, and assessment of local orientation via eigenvalue analysis of the Hessian matrix $[10,13]$.

\footnotetext{
* This research was sponsored by the Netherlands Ministry of Economic Affairs (Program IOP Beeldverwerking). We are indebted to our colleagues Dr. Theo van Walsum, Onno Wink and Joes Staal for fnuitful discussions and various contributions to the paper.
} 
The multiscale approach we discuss in this paper is inspired by the work of Sato et al. [13] and Lorenz et al. [10] who use the eigenvalues of the Hessian to determine locally the likelihood that a vessel is present. We modify their approach by considering all eigenvalues and giving the vesselness measure an intuitive, geometric interpretation. Examples on medical image data are included.

\section{Method}

In our approach we conceive vessel enhancement as a filtering process that searches for geometrical structures which can be regarded as tubular. Since vessels appear in different sizes it is important to introduce a measurement scale which varies within a certain range.

A common approach to analyze the local behavior of an image, $L$, is to consider its Taylor expansion in the neighborhood of a point $\mathbf{x}_{o}$,

$$
L\left(\mathbf{x}_{o}+\delta \mathbf{x}_{o}, s\right) \approx L\left(\mathbf{x}_{o}, s\right)+\delta \mathbf{x}_{o}^{T} \nabla_{o, s}+\delta \mathbf{x}_{o}^{T} \mathcal{H}_{o, s} \delta \mathbf{x}_{o}
$$

This expansion approximales the structure of the image up to second order. $\nabla_{o, s}$ and $\mathcal{H}_{o, s}$ are the gradient vector and Hessian matrix of the image computed in $\mathbf{x}_{o}$ at scale $s$. To calculate these differential operators of $L$ in a well-posed fashion we use concepts of linear scale space theory $[5,6]$. In this framework differentiation is defined as a convolution with derivatives of Gaussians:

$$
\frac{\partial}{\partial x} L(\mathbf{x}, s)=s^{\gamma} L(\mathbf{x}) * \frac{\partial}{\partial x} G(\mathbf{x}, s)
$$

where the $D$-dimensional Gaussian is defined as:

$$
G(\mathbf{x}, s)=\frac{1}{\sqrt{\left(2 \pi s^{2}\right)} D} e^{-\frac{\|x\|^{2}}{2 s^{2}}}
$$

The parameter $\gamma$ was introduced by Lindeberg [9] to define a family of normalized derivatives. This normalization is particularly important for a fair comparison of the response of differential operators at multiple scales. When no scale is preferred $\gamma$ should be set to unity.

Analyzing the second order information (Hessian) has an intuitive justification in the context of vessel detection. The second derivative of a Gaussian kernel at scale $s$ generates a probe kernel that measures the contrast between the regions inside and outside the range $(-s, s)$ in the direction of the derivative (figure 1$)$. This approach is the one followed in this work.
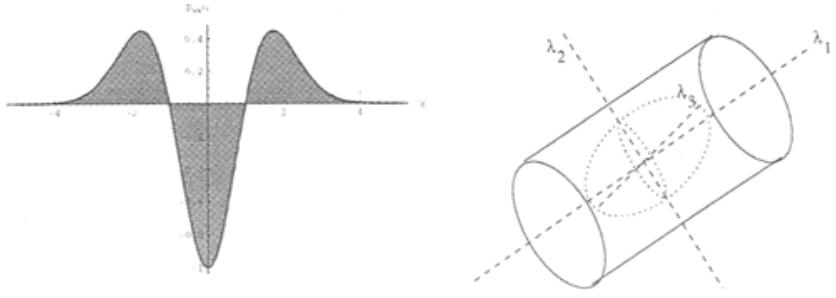

Fig. 1. Left: The second order derivative of a Gaussian kernel probes inside/outside contrast of the range $(-s, s)$. In this example $s=1$. Right: The second order ellipsoid describes the local principal directions of curvature. 
The third term in Equation (1) gives the second order directional derivative,

$$
\delta \mathbf{x}_{o}^{T} \mathcal{H}_{o, s} \delta \mathbf{x}_{o}=\left(\frac{\partial}{\partial \delta \mathbf{x}_{o}}\right)\left(\frac{\partial}{\partial \delta \mathbf{x}_{o}}\right) L\left(\mathbf{x}_{o}, s\right)
$$

The idea behind cigenvalue analysis of the Hessian is to extract the principal directions in which the local second order structure of the image can be decomposed. Since this directly gives the direction of smallest curvature (along the vessel) application of several filters in multiple orientations is avoided. This latter approach is computationally more expensive and requires a discretization of the orientation space.

Let $\lambda_{s, k}$ denote the eigenvalue corresponding to the $\mathrm{k}$-th normalized eigenvector $\hat{\mathbf{u}}_{s, k}$ of the Hessian $\mathcal{H}_{o, s}$, all computed at scale $s$. From the definition of eigenvalues:

$$
\mathcal{H}_{o, s} \hat{\mathbf{u}}_{s, k}=\lambda_{s, k} \hat{\mathbf{u}}_{s, k}
$$

and it follows that

$$
\hat{\mathbf{u}}_{s, k}^{T} \mathcal{H}_{o, s} \hat{\mathbf{u}}_{s, k}=\lambda_{s, k}
$$

By analyzing Equations (4)-(6) a nice geometric interpretation arises. The eigenvalue decomposition extracts three orthonormal directions which are invariant up to a scaling factor when mapped by the Hessian matrix. In particular, a spherical neighborhood centered at $\mathbf{x}_{0}$ with radius $1, \mathcal{N}_{x_{0}}$, will be mapped by $\mathcal{H}_{o}$ onto an ellipsoid whose axes are along the directions given by the eigenvectors of the Hessian and the corresponding axis' semi-lengths are the magnitudes of the respective eigenvalues.

This ellipsoid locally describes the second order structure of the image (thus we coin it second order ellipsoid-figure 1-) and can be used as an intuitive tool for the design of geometric similarity measures.

In the remainder of the paper $\lambda_{k}$ will be the eigenvalue with the $\mathrm{k}$-th smallest magnitude $\left(\left|\lambda_{1}\right| \leq\left|\lambda_{2}\right| \leq\left|\lambda_{3}\right|\right)$. Under this assumption Table 1 summarizes the relations that must hold between the eigenvalues of the Hessian for the detection of different structures. In particular, a pixel belonging to a vessel region will be signaled by $\lambda_{1}$ being small (ideally zero), and $\lambda_{2}$ and $\lambda_{3}$ of a large magnitude and equal sign (the sign is an indicator of brightness/darkness). The respective eigenvectors point out singular directions: $\hat{\mathbf{u}}_{1}$ indicates the direction along the vessel (minimum intensity variation) and $\hat{\mathbf{u}}_{2}$ and $\hat{\mathbf{u}}_{3}$ form a base for the orthogonal plane. We are interested in "vesselness" measures suited for medical images. In MRA and CTA, vessels emerge as bright tubular structures in a darker environment. This prior information related to the imaging modality can be used as a consistency check to discard structures present in the dataset with a polarity different than the one sought. Accordingly, we shall look for structures whose $\lambda_{2}$ and $\lambda_{3}$ are both simultaneously negative.

To summarize, for an ideal tubular structure in a $3 D$ image:

$$
\begin{aligned}
\left|\lambda_{1}\right| & \approx 0 \\
\left|\lambda_{1}\right| & \ll\left|\lambda_{2}\right| \\
\lambda_{2} & \approx \lambda_{3}
\end{aligned}
$$

and the sign of $\lambda_{2}$ and $\lambda_{3}$ indicate its polarity.

We emphasize that all three eigenvalues play an important role in the discrimination of the local orientation pattern. This will yield expressions that differ from the similarity measures proposed by Sato et al. [13] and Lorenz et al. [10] who only make use of two 
eigenvalues in their respective $3 D$ line filters. In particular, Sato's approach [13] uses a different eigenvalue ordering scheme: they are sorted in increasing value (not absolute value), and only the two largest are considered in the line filter. This implies that dark and bright lines are not treated in a similar manner.

Our dissimilarity measure takes into account two geometric ratios based on the second order ellipsoid. The first ratio accounts for the deviation from a blob-like structure but cannot distinguish between a line- and a plate-like pattern:

$$
\mathcal{R}_{\mathcal{B}}=\frac{\text { Volume } /(4 \pi / 3)}{(\text { Largest Cross Section Area } / \pi)^{3 / 2}}=\frac{\left|\lambda_{1}\right|}{\sqrt{\left|\lambda_{2} \lambda_{3}\right|}}
$$

This ratio attains its maximum for a blob-like structure and is zero whenever $\lambda_{1} \approx 0$, or $\lambda_{1}$ and $\lambda_{2}$ tend to vanish (notice that $\lambda_{1} / \lambda_{2}$ remains bounded even when the second eigenvalue is very small since its magnitude is always larger than the first).

The second ratio refers to the largest area cross section of the ellipsoid (in the plane orthogonal to $\hat{\mathbf{u}}_{1}$ ) and accounts for the aspect ratio of the two largest second order derivatives. This ratio is essential for distinguishing between plate-like and line-like structures since only in the latter case it will be zero,

$$
\mathcal{R}_{\mathcal{A}}=\frac{\text { (Largest Cross Section Area) } / \pi}{\text { (Largest Axis Semi-length) }^{2}}=\frac{\left|\lambda_{2}\right|}{\left|\lambda_{3}\right|}
$$

The two geometric ratios we introduced so far are grey-level invariant (i.e., they remain constant under intensity re-scalings). This ensures that our measures only capture the geometric information of the image. However, in MRA and CTA images there is additional knowledge available: vessel structures are brighter than the background and occupy a (relatively) small volume of the whole dataset. If this information is not incorporated background pixels would produce an unpredictable filter response due to random noise fluctuations. However, a distinguishing property of background pixels is that the magnitude of the derivatives (and thus the eigenvalues) is small, at least for typical signal-to-noise ratios present in acquired datasets. To quantify this we propose the use of the norm of the Hessian. We use the Frobenius matrix norm since it has a simple expression in terms of the eigenvalues when the matrix is real and symmetric. Hence we define the following measure

\begin{tabular}{|c|c|c|c|c|c|}
\hline $2 D$ & \multicolumn{2}{|c|}{$3 D$} & orientation paltern \\
\hline \hline$\lambda_{1}$ & $\lambda_{2}$ & $\lambda_{1}$ & $\lambda_{2}$ & $\lambda_{3}$ & \\
\hline $\mathrm{N}$ & $\mathrm{N}$ & $\mathrm{N}$ & $\mathrm{N}$ & $\mathrm{N}$ & nolsy, no preferred direction \\
\hline & & $\mathrm{L}$ & $\mathrm{L}$ & $\mathrm{H}-$ & plate-like structure (bright) \\
\hline & & $\mathrm{L}$ & $\mathrm{L}$ & $\mathrm{H}+$ & plate-like structure (dark) \\
\hline $\mathrm{L}$ & $\mathrm{H}-$ & $\mathrm{L}$ & $\mathrm{H}-$ & $\mathrm{H}-$ & tubular structure (bright) \\
\hline $\mathrm{L}$ & $\mathrm{H}+$ & $\mathrm{L}$ & $\mathrm{H}+$ & $\mathrm{H}+$ & tubular structure (dark) \\
\hline $\mathrm{H}-$ & $\mathrm{H}-$ & $\mathrm{H}-$ & $\mathrm{H}-$ & $\mathrm{H}-$ & blob-like structure (bright) \\
\hline $\mathrm{H}+$ & $\mathrm{H}+$ & $\mathrm{H}+$ & $\mathrm{H}+$ & $\mathrm{H}+$ & blob-like structure (dark) \\
\hline
\end{tabular}

Table 1. Possible patterns in $2 D$ and $3 D$, depending on the value of the eigenvalues $\lambda_{k}$ $(\mathrm{H}=$ high, $\mathrm{L}=$ low, $\mathrm{N}=$ noisy, usually small, $+/$ - indicate the sign of the eigenvalue). The eigenvalues are ordered: $\left|\lambda_{1}\right| \leq\left|\lambda_{2}\right| \leq\left|\lambda_{3}\right|$. 
of "second order structureness",

$$
\mathcal{S}=\|\mathcal{H}\|_{F}=\sqrt{\sum_{j \leq D} \lambda_{j}^{2}}
$$

where $D$ is the dimension of the image.

This measure will be low in the background where no structure is present and the eigenvalues are small for the lack of contrast. In regions with high contrast compared to the background, the norm will become larger since at least one of the eigenvalues will be large. We therefore propose the following combination of the components to define a vesselness function,

$$
\mathcal{V}_{o}(s)= \begin{cases}0 & \text { if } \lambda_{2}>0 \text { or } \lambda_{3}>0 \\ \left(1-\exp \left(-\frac{\mathcal{R}_{A^{2}}^{2}}{2 \alpha^{2}}\right)\right) \exp \left(-\frac{\mathcal{R}_{a^{2}}}{2 \beta^{2}}\right)\left(1-\exp \left(-\frac{\mathcal{S}^{2}}{2 c^{2}}\right)\right) & \end{cases}
$$

where $\alpha, \beta$ and $c$ are thresholds which control the sensitivity of the line filter to the mensures $\mathcal{R}_{\mathcal{A}}, \mathcal{R}_{\mathcal{B}}$ and $\mathcal{S}$. The idea behind this expression is to map the features in Equations (10)-(12) into probability-like estimates of vesselness according to different criteria. We combine the different criteria using their product to ensure that the response of the filter is maximal only if all three criteria are fulfilled. In all the results presented in this work $\alpha$ and $\beta$ were fixed to 0.5 . The value of the threshold $c$ depends on the grey-scale range of the image and half the value of the maximum Hessian norm has proven to work in most cases. However, future research will be directed towards automating this threshold selection. We expect that this threshold can be fixed for a given application where images are routinely acquired according to a standard protocol.

The vesselness measure in Equation (13) is analyzed at different scales, $s$. The response of the line filter will be maximum at a scale that approximately matches the size of the vessel to detect. We integrate the vesselness measure provided by the filter response at different scales to obtain a final estimate of vesselness:

$$
\mathcal{V}_{o}(\gamma)=\max _{s_{\min } \leq s \leq s_{\max }} \mathcal{V}_{o}(s, \gamma)
$$

where $s_{\min }$ and $s_{\max }$ are the maximum and minimum scales at which relevant structures are expected to be found. They can be chosen so that they will cover the range of vessel widths.

For $2 D$ images we propose the following vesselness measure which follows from the same reasoning as in $3 D$,

$$
V_{o}(s)= \begin{cases}0 & \text { if } \lambda_{2}>0 \\ \exp \left(-\frac{\mathcal{R}_{g^{2}}}{2 \beta^{2}}\right)\left(1-\exp \left(-\frac{\mathcal{S}^{2}}{2 c^{2}}\right)\right) & \end{cases}
$$

Here, $\mathcal{R}_{\mathcal{B}}=\lambda_{1} / \lambda_{2}$ is the blobness measure in $2 D$ and accounts for the eccentricity of the second order ellipse.

Equations (13) and (15) are given for bright curvilinear structures (MRA and CTA). For dark objects (as in DSA) the conditions (or the images) should be reversed. 


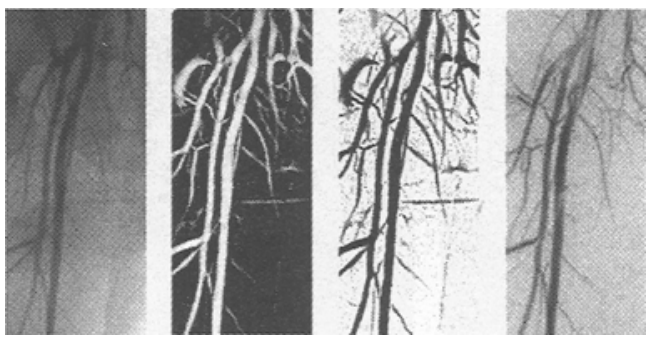

Fig. 2. Left: Part of a contrast X-ray image of the peripheral vasculature. Middle-left: Calculated vesselness of the left image. Middle-right: Calculated vesselness after inversion of the grey-scale map. Right: Image obtained by subtracting reference (without contrast) image from left image; shown here to facilitate visual inspection of the results of the filtering procedure.

\section{Results}

\subsection{D DSA images}

In this section we show some results of vessel enhancement filtering in 2D DSA images. These images are obtained by acquiring an $\mathrm{X}$-ray projection when intra-arterial contrast material is injected. A reference image is first acquired without contrast, which is subtracted from the image with contrast for background suppression. If no motion artifacts are present the subtracted images are of such good quality, that further processing is not desirable. We therefore only apply our enhancement filter to the contrast images directly, and use the subtracted images to be able to judge the performance of the vessel enhancement filter.

In figure 2, a part of an image of the peripheral vasculature is shown, where performance of subtraction is usually quite good. Although contrast is not very high in the contrast images, the method detects most vessels, over a large size range. Notice however that some artifacts where introduced in regions where background fluctuations have line patterns.

\subsection{D MRA images}

We have applied our method to three-dimensional aortoiliac MRA datasets, to show the potential of enhancement filtering to improve visualization of the vasculature. In figure 3 (left) we show a maximum intensity projection which is applied directly to the greyscale data of an MRA dataset of the aortoiliac arteries. By determining the vesselness of the MRA image at multiple scales we obtain separate images depicting vessels of various widths. This is shown in figure 4 . Here we plotted maximum intensity projections of the vesselness at four scales. The rightmost image shows how we can combine these multiscale measurements by using a scale selection procedure (recall that we work with normalized derivatives), eventually yielding a display of both the small and large vessels. The figure also shows that small and large vessels can be distinguished, which can for example be used in artery/vein segmentation. Since the enhancement filtering does not give a high output at other structures, additional information can more easily be visualized. In the middle frame of figure 3 we show the maximum intensity projection which is obtained after vessel enhancement filtering. In the right frame a closest vessel projection is shown. In this case, it is possible to determine the order in depth of various vascular structures. The excellent noise and background suppression provided by the vesselness measure greatly facilitates the use 

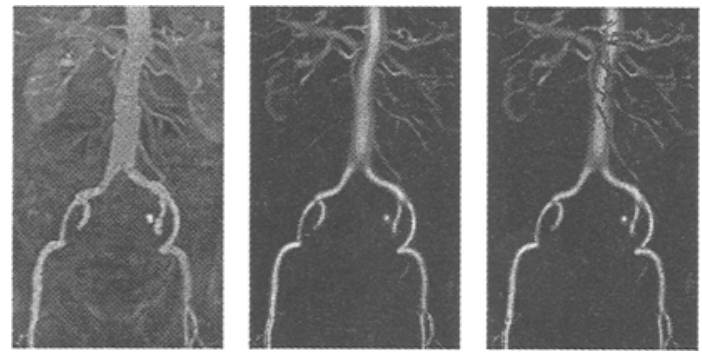

Fig. 3. Left: Original maximum intensity projection of a contrast (Gd-DTPA) MRA image. Middle: Maximum intensity projection of vessel enhanced image. We obtain quite good background suppression. Right: Closest vessel projection, facilitated by the filter's excellent background suppression.
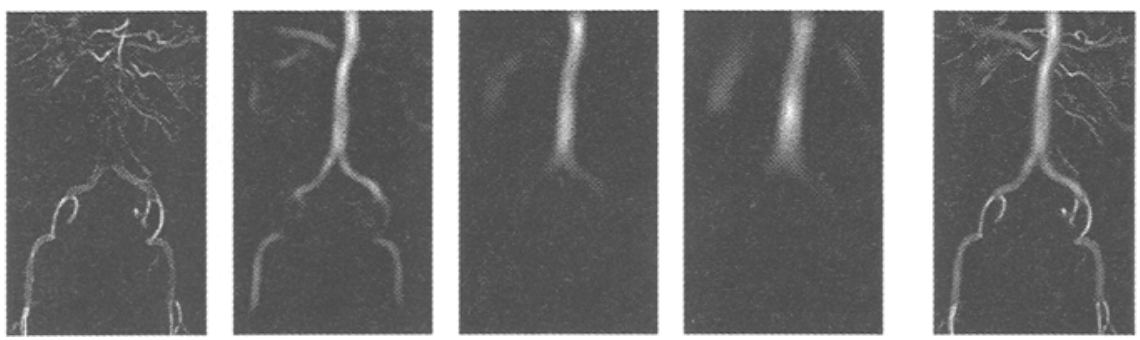

Fig. 4. The first four images show the vesselness obtained at increasing scales. The last image is the result after the scale selection procedure.

of a closest vessel projection. In order to compare the results of the vessel enhancement procedure with renderings obtained using a threshold on the original image, we show both renderings in figure 5 . We see that the original image has more background disturbance. However, the vessels tend to be narrower in the vessel enhancement image compared to the original dataset. This is due to the fact that at the boundaries of vessels the vesselness is not very high. The vessel enhancement filtering should be used in a subsequent segmentation procedure for obtaining quantitative measurements on the vasculature.

\section{Discussion}

We have presented a method for vessel enhancement filtering which is based on local structure. To this end we examined the local second order ellipsoid. Since we use information about all axes of the second order ellipsoid (all eigenvalues), the approach is a generalization of other existing approaches on the use of second order information for line detection. Recently, Sato et al. [13] and Lorenz et al. [10] used eigenvalue analysis of the Hessian for vessel enhancement, but they did not use all eigenvalues simultaneously. We have shown the excellent noise and background suppression in a two clinical image modalities, underlying the potential of the approach.

It is important to realize that we do not obtain a segmentation of the vasculature. Only if an accurate model of the typical luminance in the perpendicular direction of the vessel is 
known, an estimate of the size of the vessel can be made based on the response of the filter over scales. However this is often not the case. For example, in MRI it is common to reduce the reconstruction time by restricting the number of lines in $k$-space (scan percentage) which accounts for a reduction of the effective Fourier spectrum of the measured signal. This technique can lead to ringing artifacts (overshoot) in high transition steps (for example, in vessels boundaries) thus violating simplified profile models (Gaussian/bar-like $[7,10]$ ). The vesselness measure can serve as a preprocessing step for segmentation of this type of images.

\section{References}

1. S. Aylward, et al. . Intensity ridge and widths for tubular object segmentation and description. In A. A. Amini, F. L. Bookstein, and D. C. Wilson, eds., Math. Meth. in Biomed. Imag. Anal, pages 131-138, 1995.

2. H. Chen and J. Hale. An algorithm for MR angiography image enhancement. $M R M, 33(4): 534$ 40, April 1995.

3. Y. P. Du and D. L. Parker. Vessel enhancement filtering in three-dimensional MR angiography. JMRI, 5(3):353-359, 1995.

4. Y. P. Du and D. L. Parker. Vessel enhancement filtering in three-dimensional MR angiograms using long range signal correlation. $J M R I, 7(2): 447-450,1997$.

5. L. M. J. Florack, et al. Scale and the differential structure of images. Imag. and Vis. Comp., 10(6):376-388, July/August 1992.

6. J. J. Koenderink. The structure of images. Biol. Cybern., 50:363-370, 1984.

7. T. M. Koller, et al. Multiscale detection of curvilinear structures in 2-D and 3-D image data. In E. Grimson, S. Shafer, A. Blake, and K. Sugihara, editors, Proc. Fifth Int. Conf. on Comp. Vis., pages 864-869, 1995.

8. T. M. Koller. From Data to Information: Segmentation, Description and Analysis of the Cerebral Vascularity. Diss. ETH no. 11367, Swiss Federal Institute of Technology ETHZ, 1995.

9. T. Lindeberg. Edge detection and ridge detection with automatic scale selection. In Proc. Conf. on Comp. Vis. and Pat. Recog., pages 465-470, San Francisco, CA, June 1996.

10. C. Lorenz, et al.. Multi-scale line segmentation with automatic estimation of width, contrast and tangential direction in 2D and 3D medical images. In J. Troccaz, E. Grimson, and R. Mösges, eds., Proc. CVRMed-MRCAS'97, LNCS, pages 233-242, 1997.

11. M. M. Orkisz, et al. Improved vessel visualization in MR angiography by nonlinear anisotropic filtering. $M R M, 37(6): 914-9$, June 1997.

12. M. R. Prince. Gadolinium-enhanced MR aortography. Radiol., 191:155-164, 1994.

13. Y. Sato, et al. 3D multi-scale line filter for segmentation and visualization of curvilinear structures in medical images. In J. Troccaz, E. Grimson, and R. Mosges, eds., Proc. CVRMedMRCAS'97, LNCS, pages 213-222, 1997.
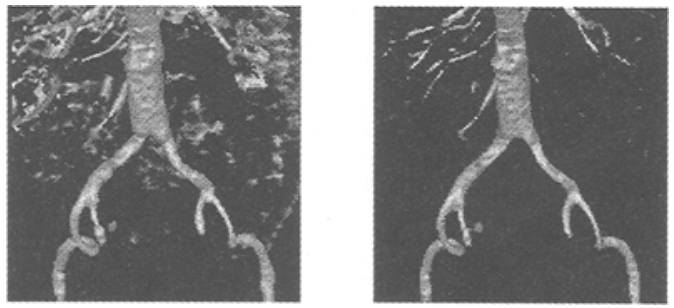

Fig. 5. Left: Volume rendering based on threshold of the original dataset. Right: Volume rendering based on threshold of the vesselness image. 